$>$ Le rôle des cellules dendritiques dans l'asthme et la rhinite allergique est établi depuis 15 ans. Dans cette synthèse, nous résumons les principales découvertes concernant la fonction des cellules dendritiques dans l'allergie respiratoire, en nous concentrant sur les études réalisées chez l'homme. Outre une expression importante du récepteur de haute affinité à l'IgE (immunoglobuline $\varepsilon$ ), les cellules dendritiques de patients allergiques sont caractérisées par une propension à induire des réponses allergiques en réaction à une stimulation par les allergènes et/ou certaines cytokines épithéliales. Les données mécanistiques concernant cette dérégulation des cellules dendritiques dans l'asthme offrent des perspectives thérapeutiques innovantes. <

Les maladies allergiques sont en pleine expansion. Ainsi, l'asthme, maladie allergique dans 60 à $70 \%$ des cas, touche environ 300 millions de personnes dans le monde et ce nombre devrait encore progresser pour atteindre 400 millions d'ici 2025 [1]. Quant à la rhinite allergique (RA), elle touche près de 500 millions de personnes [2]. Les principaux facteurs évoqués pour expliquer cette augmentation d'incidence de l'asthme allergique (AA) sont la pollution atmosphérique, le regroupement des populations dans les villes, ainsi que le mode de vie occidental [1]. D'un point de vue immunologique, les allergies sont caractérisées par une réponse inflammatoire inappropriée aux antigènes de l'environnement [3]. Cette réponse est principalement de type Th2, mais d'autres sous-classes de lymphocytes sont impliquées, y compris les lymphocytes Th9 et Th17 [4, 5]. Les cellules dendritiques (CD) sont des cellules spécialisées dans la présentation d'antigènes aux cellules responsables de l'immunité adaptative $[6,53]$. On les retrouve à la surface de toutes les muqueuses, dans certains organes solides, au sein des organes lymphoïdes secondaires et primaires, ainsi que dans le sang. Au croisement entre

\section{Les cellules dendritiques humaines dans l'asthme et la rhinite allergique}

Antoine Froidure ${ }^{1}$, Charles Pilette ${ }^{1,2}$

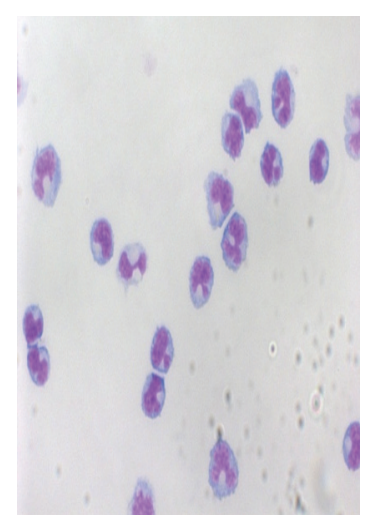

${ }^{1}$ Institut de recherche expérimentale et clinique, Université catholique de Louvain, Walloon institute for excellence in lifesciences and biotechnology (WELBIO), avenue Hippocrate 54 , boîte 1.54.04, 1200 Bruxelles, Belgique ;

${ }^{2}$ Cliniques universitaires Saint-Luc, service de pneumologie, Bruxelles, Belgique. antoine.froidure@uclouvain.be

immunités innée et adaptative, les CD déclenchent les réponses immunitaires. En plus de présenter les antigènes avec le complexe majeur d'histocompatibilité ( ${ }^{\text {er }}$ signal), ces cellules ont la capacité d'influencer la réponse immunitaire via l'expression de molécules de costimulation ( $2^{\mathrm{e}}$ signal) et la sécrétion de cytokines ( $3^{\mathrm{e}}$ signal) (Figure 1) [6]. Comme les CD ont la capacité d'induire et de polariser les réponses immunitaires, l'étude de leur fonction chez les patients allergiques est d'un grand intérêt. Les recherches dans ce domaine n'ont cessé d'évoluer depuis 15 ans. Dans cet article, nous brossons un tableau de l'état actuel des connaissances concernant la biologie des CD dans l'asthme et la rhinite allergiques. Nous revenons également sur les relations entre les CD et leur environnement, ainsi que sur les perspectives thérapeutiques dévoilées par leur physiopathologie.

\section{Classification et nomenclature des cellules dendritiques}

Les CD sont divisées en plusieurs sous-types distincts. L'identification des différentes classes de CD a d'abord été faite chez la souris [7], puis chez l'homme [8]. Le Tableau / fait la liste des différents soustypes de CD avec leurs principaux marqueurs de surface.

- Chez la souris, on en distingue trois types : (1) les CD conventionnelles (cCD), elles-mêmes divisées en deux populations distinctes exprimant soit CDllb, soit CD103 [6] ; (2) les CD plasmacytoïdes (pCD), qui sont issues d'un progéniteur différent de celui des CD conventionnelles; elles sont caractérisées par une morphologie rappelant celle des plasmocytes [9] et par leur capacité à sécréter de grandes quantités d'interféron- $\alpha$ $($ IFN- $\alpha$ ) lors des réponses antivirales; et (3), les CD inflammatoires 


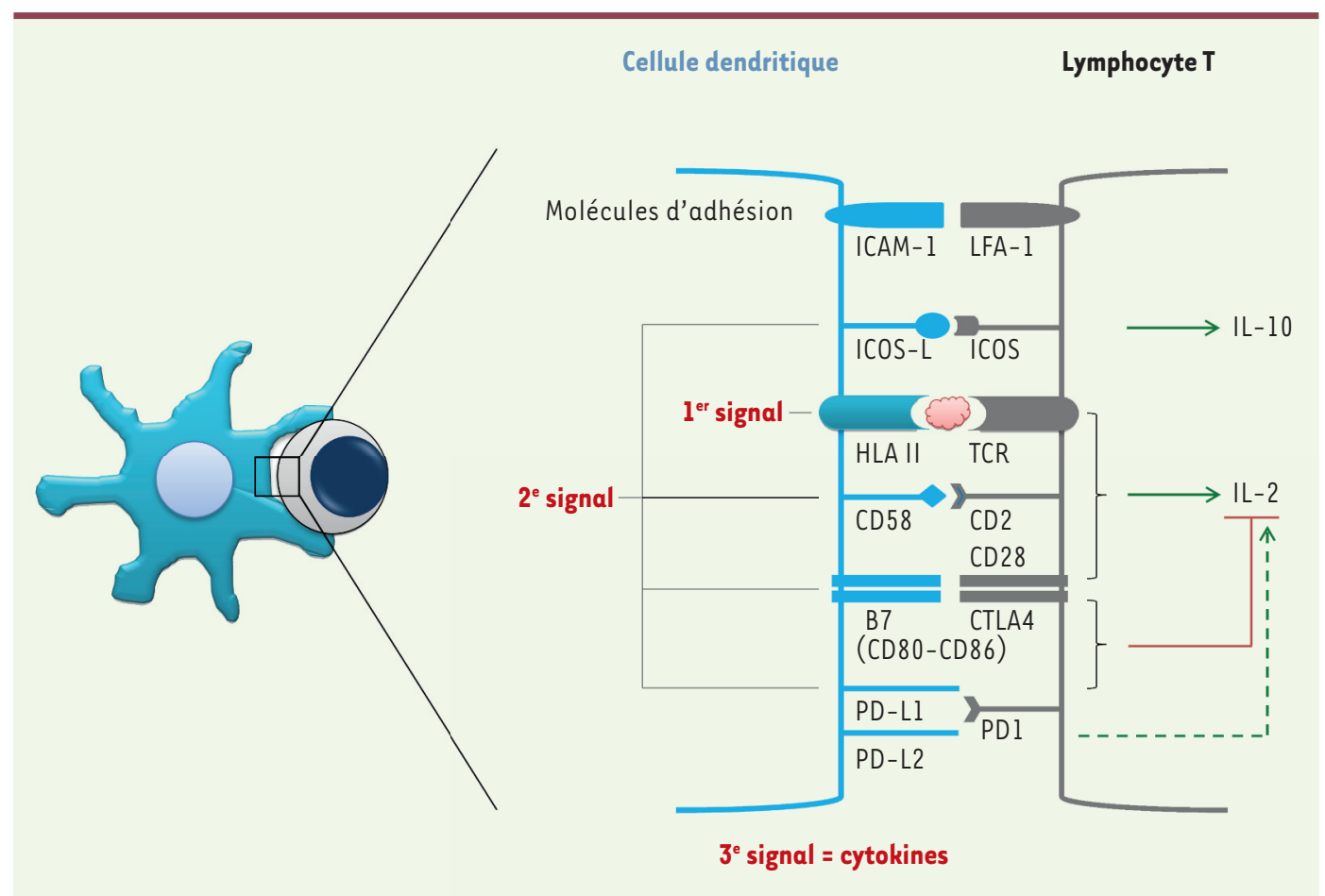

Figure 1. Principales molécules d'interactions entre cellules dendritiques et lymphocytes $T$ lors de la présentation d'un antigène/ allergène. Le $1^{\text {er }}$ signal, qui confère la spécificité, est fourni par la présentation de l'antigène via les molécules HLA de type 2. Des molécules d'adhésion (ICAM-1 [intercellular adhesion molecule-1], LFA-1 [lymphocyte function-associated antigen-1]) renforcent le lien entre les cellules. Le $2^{\mathrm{e}}$ signal est constitué par l'interaction des molécules de costimulation (ICOS-L [inducible co-stimulator], PD-L1/2 [programmed death-ligand 1/2], CD86, CD83). Enfin, un $3^{e}$ signal potentiel consiste en la liaison de certaines cytokines avec leur récepteur à la surface des lymphocytes $T$.

(infCD) qui sont recrutées in situ à partir des monocytes lors d'une agression. Ces CD ont une durée de vie courte, migrent peu et sont, chez la souris, les seules à exprimer le récepteur de haute affinité à l'immunoglobuline ( $\mathrm{g} g) \varepsilon(F c \varepsilon R I)$. Elles expriment également certains marqueurs monocytaires à leur surface $[10,11]$ (Tableau I).

- Chez l'homme, on distingue les CD conventionnelles myéloïdes $(m C D)$ et plasmacytoïdes ( $p C D)$. Les CD myéloïdes sont encore divisées en un sous-type majoritaire $(m C D l)$ et une petite population (mCD2), exprimant BDCAl (CDlc) (blood dendritic cells antigen 1 ) et BDCA3 (CD141), respectivement [54]. II faut également signaler la présence, au sein de la muqueuse respiratoire, d'un sous-type de cellules dendritiques exprimant le CD la et la langerine, rappelant les cellules de Langerhans cutanées [12, 13, 55]. L'équivalent humain des $C D$ inflammatoires de la souris a été mis en évidence pour la $l^{\text {re }}$ fois en 2013 [14, 56], dans le liquide synovial de patients souffrant de polyarthrite rhumatoïde, ainsi que dans l'ascite de patientes atteintes d'un cancer ovarien. Ces cellules ont un génotype et un phénotype intermédiaires entre ceux de monocytes et de CD conventionnelles myéloïdes et ont la capacité d'induire une réponse Th17. À ce jour, elles n'ont pas été clairement identifiées dans le poumon ou la muqueuse nasale.

II faut signaler également que des CD dérivées in vitro des monocytes (moCD) sont largement utilisées en recherche. Elles constituent un substitut aux CD conventionnelles myéloïdes, mais partagent quelques caractéristiques avec les CD inflammatoires murines. II est important de noter que ces $C D$ dérivées in vitro des monocytes se comportent différemment des $C D$ conventionnelles myéloïdes in vitro, notamment concernant leur capacité d'endocytose, qui est moindre que celle de ces dernières [15]. Elles n'ont donc pas de parfait équivalent in vivo.

\section{Implication des cellules dendritiques dans l'asthme et la rhinite allergique}

Les premières preuves de l'implication des CD dans l'asthme ont été apportées par l'équipe de Bart Lambrecht qui montra, en 1998, que les CD conventionnelles étaient indispensables au déclenchement de l'allergie chez la souris sensibilisée à l'ovalbumine (OVA) [16]. Toujours chez la souris, il a été démontré, en 2004, que les CD plasmacytoïdes avaient un rôle protecteur et tolérogène vis-à-vis des allergènes [17]. Récemment, l'implication des CD conventionnelles $C D 11 b^{+}$et des CD inflammatoires dans l'asthme a été mise en évidence chez la souris, bien que des zones d'ombre persistent: les premières $\left(\mathrm{CD} l \mathrm{lb}^{+}\right)$semblent essentielles à l'induction de l'immunité Th2 dans les ganglions lymphatiques régionaux, alors que les secondes, dérivées des monocytes, déclenchent la réaction allergique au niveau local [11]. Les CD conventionnelles $C D 103^{+}$, quant à elles, jouent un rôle dans la présentation d'antigènes 


\begin{tabular}{|c|c|}
\hline CD murines & CD humaines \\
\hline $\begin{array}{l}\text { CD1lb }^{+} \text {cDC } \\
\text { CD1lc, CD11b, MHCII, CD } 4 \S, C D 8 \alpha^{\S}, T L R 1,2,3,4,5,6,9\end{array}$ & 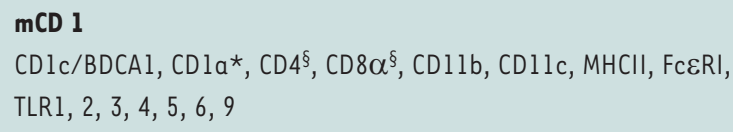 \\
\hline $\begin{array}{l}\mathrm{CD} \mathbf{O 3}^{+} \mathrm{CDC} \\
\mathrm{CD} 1 \mathrm{c}, \mathrm{CD} 1 \mathrm{lc}, \mathrm{CD} 103 \text {, langerine, MHCII, TLRI, 2, 3, 4, 5, 6, } 9\end{array}$ & $\begin{array}{l}\text { mCD } 2 \\
\text { CD14l/BDCA3, CDIlc, MHCII, CLEC9A, XCR1, TLR3 } \\
\text { CD pulmonaires CDla+ CDlc- } \\
\text { CDla, langerine, MHCII }\end{array}$ \\
\hline $\begin{array}{l}\text { PCD } \\
\text { MHCII, CD123 (IL-3R } \alpha), C D 4, \text { Ly6C, TLR7, } 9\end{array}$ & $\begin{array}{l}\text { pCD } \\
\text { MHCII, BDCA2, BDCA4, CD123 (IL-3R } \alpha), \text { CD4, FcERI, Ly6C } \\
\text { TLR7, } 9\end{array}$ \\
\hline $\begin{array}{l}\text { infCD } \\
\text { MHCII, CDIlb, CD1lc, F4/80, CD } 64 \text {, Ly6C, FceRI }\end{array}$ & $\begin{array}{l}\text { infCD } \\
\text { MHCII, CD lc/BDCAl, CDla, CD 1lb, FceRI, CD206, CD172a, CD 14, } \\
\text { TLRl, 2, 3, 4, 5, 6, } 9 \\
\text { moCD } \\
\text { HLA-DR, CDla, CD1lc, CD } 83\end{array}$ \\
\hline
\end{tabular}

Tableau I. Principaux sous-types de cellules dendritiques et leurs marqueurs de différenciation. *Une sous-population de mCDI pulmonaires exprime le CDla. ${ }^{\S}$ On retrouve dans les organes lymphoïdes des CD immatures exprimant soit le CD4, soit le CD8 $\alpha$. BDCA4: neuropilin-1; cCD : cellule dendritique conventionnelle; $C L E C$ : C-type lectin-like protein; infCD : cellule dendritique inflammatoire ; $m C D$ : cellule dendritique myéloïde ; MHC : major histocompatibility complex; moDC : cellule dendritique dérivée du monocyte ; $P C D$ : cellule dendritique plasmacytoïde ; XCR : C-subfamily of chemokine receptor.

viraux [13], mais semblent également capables, après exposition à des doses importantes d'allergènes Der $p^{1}$ de présenter les allergènes [11] et d'induire des réponses immunitaires Th2 ex vivo [18].

Chez l'homme, en 2001 et 2003, deux études ont montré que les moCD et les mCD de sujets asthmatiques stimulées avec de l'extrait d'allergène Der $p$ induisent la sécrétion d'interleukine (IL)-4 par les lymphocytes T. En outre, les CD de patients allergiques ont un défaut de sécrétion d'IL-10 et d'IL-12 en réponse à une stimulation par du lipopolysaccharide (LPS) [19, 20]. Le nombre de mCD dans le sang et les voies aériennes est plus important chez les patients avec un asthme allergique que chez les sujets sains. À l'inverse, le nombre de pCD circulantes semble plus bas chez les patients allergiques [21]. Plusieurs études ont montré que le nombre de mCD circulantes diminuait concomitamment à une augmentation des mCD dans le sputum, 6 à $8 \mathrm{~h}$ après exposition à l'allergène, ce qui conforte l'hypothèse d'un recrutement des mCD depuis le sang vers les poumons [21-23]. Enfin, il a été récemment décrit que les patients allergiques avaient moins de mCD2 circulantes que les patients contrôles [24]. La même équipe a également mis en évidence une augmentation des mCD2 pulmonaires après exposition allergénique, suggérant un rôle de ces cellules dans la réponse allergique [25]. Le phénotype des CD est également modifié chez les patients souffrant de rhinite allergique et/ou asthme allergique, avec

${ }^{1}$ L'allergène Der p fut le premier allergène découvert chez l'acarien Dermatophagoides pteronyssinus. L'allergène Der $\mathrm{p} l$ est une glycoprotéine de $25-\mathrm{kDa}$, majoritaire dans les extraits d'acariens, et capable d'induire la production d'Ig $\varepsilon$ chez $80 \%$ des personnes sensibilisées aux acariens. notamment, contrairement aux CD des sujets contrôles, une expression du FcERI à la surface de leurs mCD et de leurs PCD [26] (Figure 2). Cette expression du récepteur à l'lgદ est utilisée par les CD pour faciliter la présentation d'allergènes et amplifier la réponse lymphocytaire à de faibles concentrations d'allergènes [27], ainsi que pour améliorer la clairance des Ig circulantes [28]. En bref, l'asthme et la rhinite allergique semblent être caractérisés par une modification du nombre de cellules dendritiques, circulantes et locales, mais surtout du phénotype de ces cellules avec, en particulier, une expression du récepteur de haute affinité de l'Igદ. Notre équipe a récemment étudié, en collaboration avec le groupe de S.R. Durham (Imperial College, Londres, Royaume-Uni), le phénotype et la fonction des CD de patients souffrant de rhinite allergique aux acariens et/ou aux pollens de graminées $[29,30]$. Huit heures après exposition des patients avec rhinite allergique aux allergènes, le nombre de $\mathrm{mCD}$ et de pCD augmente dans la muqueuse nasale. Le nombre de mCD présentes dans le derme des patients augmente $48 \mathrm{~h}$ après l'injection intradermique de l'allergène. Après exposition aux allergènes, le nombre de mCD exprimant I'IL-10 double chez les sujets contrôles, ce qui n'est pas le cas pour les patients avec rhinite allergique. Chez ces derniers, on dénombre significativement moins de $P C D$ exprimant 


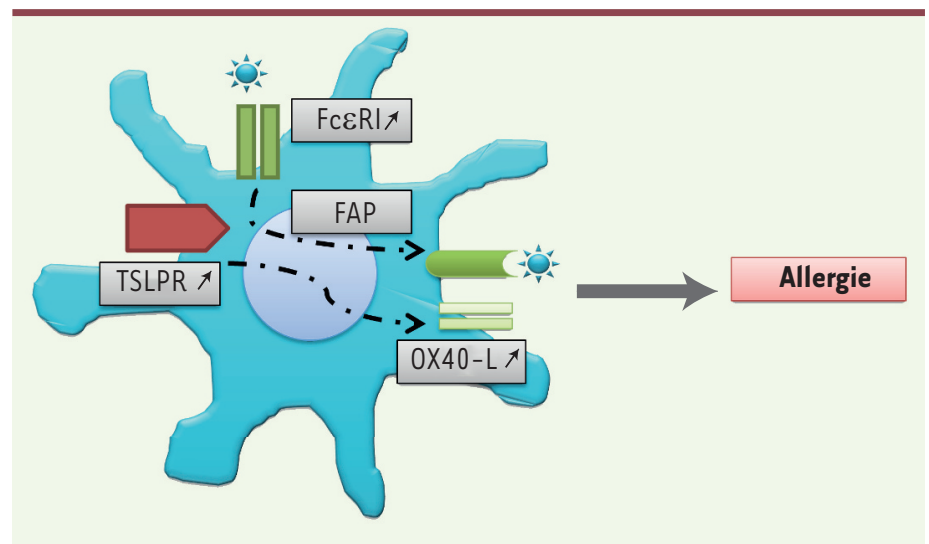

Figure 2. Caractéristiques des $m C D$ de patients allergiques. Les $m C D$ des patients allergiques ont des caractéristiques particulières: expression accrue du récepteur de haute affinité des Ig $(\mathrm{F} c \varepsilon R \mathrm{R})$, utilisé pour faciliter la présentation des antigènes (FAP pour facilitated antigen presentation) par focalisation de ceuxci à la surface des CD, ainsi que du récepteur du TSLP (TSLPR). La liaison du TSLP à son récepteur induit l'expression d'0X40-L, une molécule de costimulation impliquée dans l'induction des réponses Th2.

I'IFN- $\alpha$ que chez les patients contrôles. Enfin, lorsqu'elles sont cultivées en présence de lymphocytes $T C D 4^{+}$allogéniques, les $C D$ de patients allergiques ont la capacité d'induire la sécrétion d'IL-4 et d'IL-5 à des niveaux significativement plus élevés que les mCD de sujets contrôles. À l'inverse, I'induction d'IFN- $\gamma$ dans les lymphocytes mis en présence de CD de patients allergiques est diminuée. Cela montre qu'après exposition allergénique, on assiste à un recrutement de CD sur le site de l'inflammation. Cependant, le type de CD recrutées est fondamentalement différent chez les patients allergiques et les sujets sains : celles qui sont recrutées chez les patients ont une capacité à induire des réponses allergiques de type Th2, alors que celles qui infiltrent la muqueuse nasale des sujets contrôles expriment I'IL-10 et l'IFN- $\gamma$.

Les $C D$ expriment différentes molécules de costimulation qui servent de second signal lors de la présentation d'antigènes. L'expression relative de ces molécules permet d'orienter la différenciation des lymphocytes vers un phénotype spécifique. Ainsi, il a été démontré que les molécules de costimulation programmed death-ligand I (PD-LI) et inducible costimulator ligand (ICOS-L) étaient liées à la génération de lymphocytes T régulateurs (Treg). Nous avons analysé l'expression des molécules de costimulation de la famille $B 7$ sur les $C D$ de patients allergiques et de sujets contrôles [29] et avons montré que les $\mathrm{mCD}$ sanguines et nasales des patients ayant une rhinite allergique expriment moins d'ICOS-L que les mCD de sujets contrôles. Cultivées avec des lymphocytes $T C D 4^{+}$allogéniques, les $\mathrm{mCD}$ de patients allergiques induisent la sécrétion d'IL-13, d'IL-5 et de TNF- $\alpha$. En bloquant ICOS$L$ dans les $m C D$ de sujets contrôles non allergiques, on obtient une induction d'IL-13 similaire à celle obtenue avec les $\mathrm{MCD}$ de patients allergiques. Au contraire, le blocage de PD-Ll entraîne une induction combinée d'IL-13 et d'IFN- $\gamma$. En résumé, les mCD des patients allergiques induisent des réponses allergiques qui témoignent notamment d'une expression plus faible d'ICOS-L.

\section{GLOSSAIRE}

CD : cellule dendritique

CCD : cellule dendritique conventionnelle

$\mathrm{mCD}$ : cellule dendritique myéloïde

PCD : cellule dendritique plasmacytoïde

moDC : cellule dendritique dérivée des monocytes

infCD : cellule dendritique inflammatoire

Ig: immunoglobuline

IL : interleukine

PD-L1 : programmed death-ligand I

ICOS-L: inducible costimulation ligand

IFN : interféron

ITS : immunothérapie spécifique

TLR : toll-like receptor

TSLP : thymic stromal lymphopoietin

TSLPR : récepteur du TSLP

OX40-L : ligand d'OX40

OVA : ovalbumine

\section{Les cellules dendritiques interagissent avec l'épithélium pour initier les réponses allergiques}

Les cellules dendritiques exercent leur fonction en étroite relation avec l'épithélium bronchique et son environnement (Figure 3). Elles expriment à leur surface certains récepteurs de l'immunité innée, ce qui les rend sensibles à des stimulus non spécifiques induits par des pathogènes. Ainsi, l'allergène Der $p l$ est capable de scinder les jonctions serrées de l'épithélium bronchique et d'interagir directement avec les $C D$ [31]. L'allergène Der $p 2$ possède une structure analogue à celle du LPS bactérien; il est capable d'activer directement les cellules épithéliales et les $C D$ via le récepteur Toll-like 4 (TLR4) [32]. Après activation par les allergènes, les cellules épithéliales libèrent des cytokines qui participent au déclenchement de la réponse allergique. La thymic stromal lymphopoietin (TSLP) est un peptide de la famille de l'IL-7 qui a la propriété d'induire l'expression du ligand de $0 \times 40$ (0X40-L, une molécule de costimulation) à la surface des mCD. La liaison d' $O X 40-L$ à $0 \times 40$, qui est exprimé par les lymphocytes $T$ naïfs, polarise la réaction immunitaire vers un phénotype Th2 [33]. L'expression de TSLP est significativement plus élevée dans la muqueuse des patients asthmatiques que dans celle des sujets sains [34], mais aussi au sein de l'épithélium dans la dermatite atopique [35] et la rhinite allergique [36]. En outre, les relations entre la TSLP et les CD sont au cœur de la théorie de la «marche atopique » [37] : celle-ci fait référence à I'histoire naturelle des maladies allergiques, suivant une séquence partant de la dermatite atopique, pour ensuite mener à la rhinite allergique et l'asthme. La TSLP sécrétée 


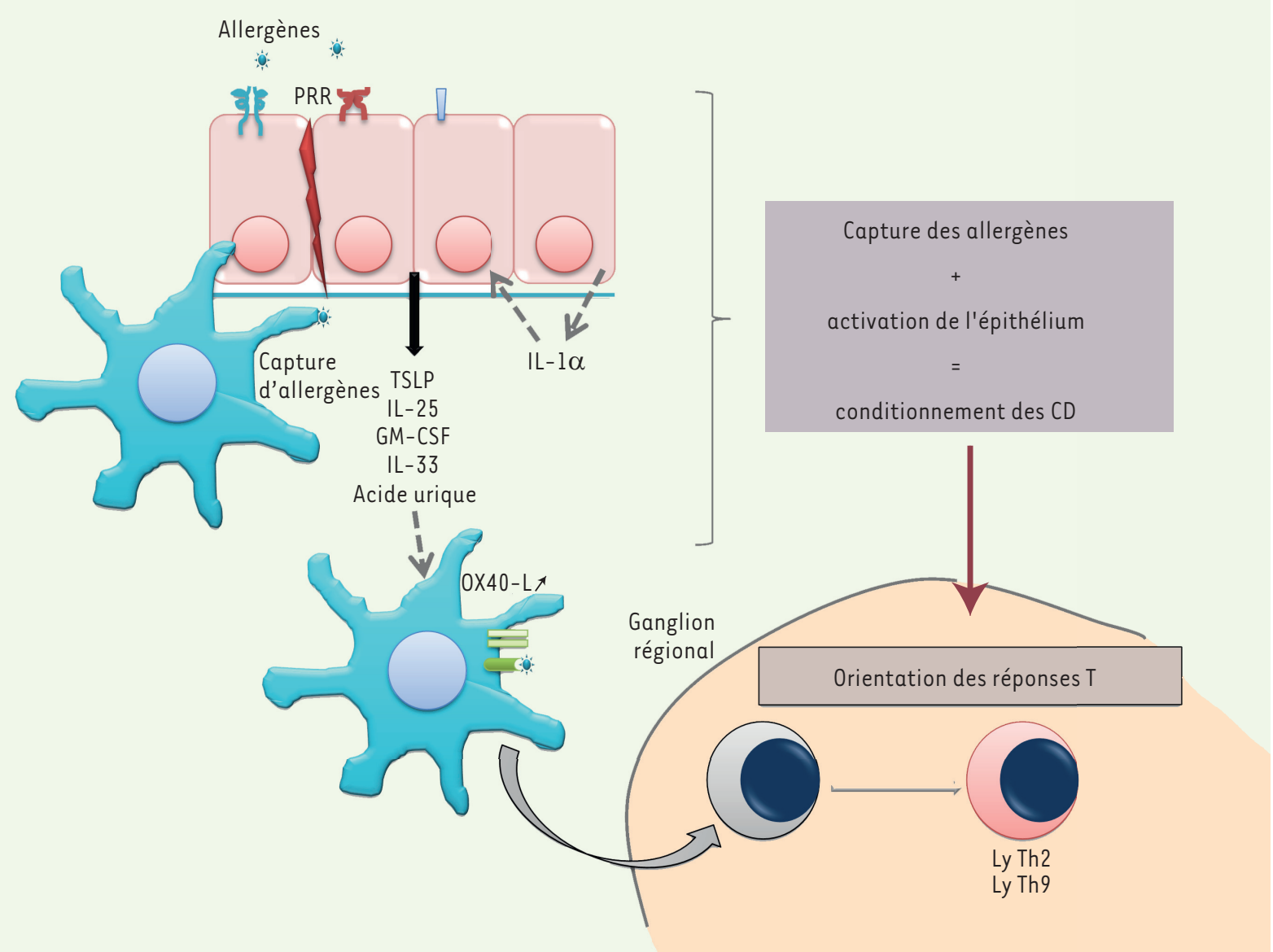

Figure 3. Les cellules dendritiques et l'épithélium bronchique interagissent pour déclencher la réaction allergique. Certains allergènes sont capables d'activer l'épithélium via les récepteurs de l'immunité innée (PRR, pattern recognition receptor) et/ou de dégrader des protéines de jonctions intercellulaires pour interagir directement avec les CD. Après activation par les allergènes, l'épithélium sécrète des cytokines (TSLP, IL-25, IL-33, GM-CSF [granulocyte-macrophage colony-stimulating factor]) qui se lient à leur récepteur à la surface des CD et des lymphocytes, entraînant une réponse immunitaire pro-allergique. Ly : lymphocyte.

au niveau cutané dans la dermatite atopique pourrait favoriser les réponses Th2 systémiques et la sensibilisation à des allergènes aéroportés $[35,37]$. Nous avons récemment mis en évidence que les patients ayant un asthme allergique ont une plus grande proportion de $\mathrm{mCD}$ exprimant le récepteur de la TSLP dans le sang et dans la muqueuse bronchique (Figure 2). Cela entraîne les mCD de ces patients à induire des réponses Th2 et Th9 après une stimulation par la TSLP [38]. De plus, l'induction de lymphocytes Th2 est dépendante du ligand d'OX40 (0X40-L), ce qui n'est pas le cas des lymphocytes Th9 dans notre étude.

De manière similaire, l'IL-33 et l'IL-25 favorisent la mise en place de réponses immunitaires allergiques, entre autres via leur action sur les CD [39] (Figure 3). L'implication de ces cytokines dans l'asthme humain n'est cependant pas aussi claire que dans les modèles murins [40]. Enfin, l'activation de l'inflammasome par les acariens et son lien avec la sécrétion d'IL-33 et de GM-CSF (granulocyte-macrophage colony stimulating factor) par l'épithélium bronchique ont été démontrés récemment par M. Willart et ses collègues dans un modèle murin in vivo, ainsi qu'in vitro en culture broncho-épithéliale [41]. Les allergènes d'acariens entraînent la sécrétion d'IL-l $\alpha$ par l'épithélium bronchique, qui stimule de manière autocrine la libération d'IL-33 et de GM-CSF, lesquels exercent leurs effets proTh2 sur leurs cellules cibles.

Des questions encore sans réponse concernent les déterminants, génétiques et/ou épigénétiques, de ces anomalies des CD dans la rhinite et l'asthme allergiques, et la contribution des modifications pathologiques de l'épithélium aux altérations fonctionnelles des cellules dendritiques.

\section{Influence des traitements anti-allergiques sur les cellules dendritiques}

L'étude des CD dévoile certaines cibles, jusqu'alors inconnues, de médications utilisées en routine dans le traitement des maladies allergiques (Tableau II). Les corticostéroïdes inhalés, pierre angulaire du traitement de l'asthme persistant [42], non seulement 


$\begin{array}{ll}\text { Corticostéroïdes (homme) } & \text { Inhibition de la maturation des CD, activation de GILZ } \\ \left.\begin{array}{ll}\text { Omalizumab (homme) } & \text { Diminution de l'expression du FcERI et inhibition des réponses Th2 in vitro } \\ \hline \text { ITS (homme) } & \text { Induction de CD tolérogènes } \\ \hline \text { Anti-TSLP (souris) } & \text { Inhibition de l'inflammation et du remodelage } \\ \text { Anti-TSLP (homme) } & \text { Diminution de la chute du VEMS après exposition allergénique } \\ \text { Anti-TSLPR (singe) } & \text { Inhibition de l'inflammation }\end{array}\right] \\ \text { Anti-ST2 (souris) } & \text { Inhibition de l'inflammation et de l'HRBNS }\end{array}$

Tableau II. Effets de traitements anti-allergiques sur les cellules dendritiques. GILZ : glucocorticoid-induced leucine zipper; ITS : immunothérapie spécifique; TSLP : thymic stromal lymphopoietin; TSLPR : récepteur du TSLP ; VEMS : volume expiré maximal en une seconde ; HRBNS : hyper-réactivité bronchique non spécifique.

diminuent significativement le nombre de CD dans les voies aériennes des asthmatiques [43], mais activent également dans les CD le promoteur GILZ (glucocorticoid-induced leucine zipper), qui favorise la transcription de gènes menant à la génération de lymphocytes T régulateurs par les CD $[44,57](\rightarrow)$. $(\rightarrow)$ Voir la Synthèse de A. Pépin et al., $\mathrm{m} / \mathrm{s}$ $n^{\circ} 1$, janvier 2015, page 75
L'omalizumab est un anticorps monoclonal dirigé contre le fragment Fc de l'IgE. II est utilisé comme traitement adjuvant de l'asthme allergique sévère [45] et diminue l'expression de surface du FcERI sur les basophiles, mais également les CD [46]. Ceci entraîne une inhibition de la présentation facilitée des allergènes et, ainsi, des réponses Th2 in vitro [47].

L'immunothérapie spécifique allergénique exerce en partie ses effets via son action sur les $C D$, en activant l'apparition de $C D$ tolérogènes, menant à l'expansion de lymphocytes T reg spécifiques [48].

\section{Les cellules dendritiques et leur environnement : futures cibles thérapeutiques?}

Étant donné le rôle central des $C D$ dans les phases initiales de la réponse allergique, les cibler est une approche prometteuse pour traiter l'allergie (Figure 4). Une expérience déjà tentée avec succès chez la souris consiste à « éduquer » les $p C D$ avec un allergène avant de les réinjecter à I'hôte. De Heer et son équipe ont ainsi montré dans un modèle de souris sensibilisées à l'ovalbumine (OVA) que les pCD préalablement stimulées par I'OVA pouvaient restaurer une tolérance vis-à-vis de l'allergène [17]. Un essai clinique récent (NCT01087515, www.clinicaltrials.gov) a étudié la fonction des CD circulantes, dans l'espoir d'utiliser les CD tolérogènes comme futur traitement des allergies.

Une autre approche, déjà largement explorée dans des modèles in vivo, est de cibler certaines cytokines dont les effets passent, entre autres, par une action sur les CD (Tableau II). Plusieurs équipes ont démontré l'efficacité dans l'asthme expérimental d'anticorps dirigés contre la
TSLP ou son récepteur $[49,50]$, ou contre le récepteur ST2 de I'IL-33 [51]. La preuve du concept ayant été confirmée chez l'homme pour la TSLP [52], des essais contrôlés devraient confirmer l'intérêt de cibler ces cytokines à l'interface entre l'épithélium et les CD en thérapeutique clinique.

\section{Conclusions et perspectives}

Depuis environ 15 ans, les preuves d'un dysfonctionnement des CD dans les maladies allergiques s'accumulent. Ces anomalies des CD de patients allergiques sont détectables in situ, mais également dans le sang de patients atopiques, c'est-à-dire ayant une prédisposition génétique aux réponses allergiques. Les $C D$ de patients allergiques sont caractérisées par une propension à induire des réponses immunes de type Th2, ainsi que Th9. De plus, l'identification de mécanismes sous-jacents, comme une surexpression du récepteur de la TSLP et un défaut d'expression d'ICOS-L, ouvre des perspectives thérapeutiques.

Certaines questions persistent; en particulier, quelle est la cause de ce dysfonctionnement des CD de patients allergiques et ou s'opère-t-il ? Une piste intéressante concerne le rôle des cytokines produites par l'épithélium respiratoire, comme le TSLP, I'IL-33, I'IL-25, l'IL-1 et le GM-CSF, en réponse à l'exposition aux allergènes. D'autres signaux provenant du microenvironnement des $C D$ dans les muqueuses, ainsi que divers polymorphismes génétiques au niveau systémique, contribuent aussi probablement à la programmation anormale des CD de patients avec asthme ou rhinite allergique. $\diamond$ 


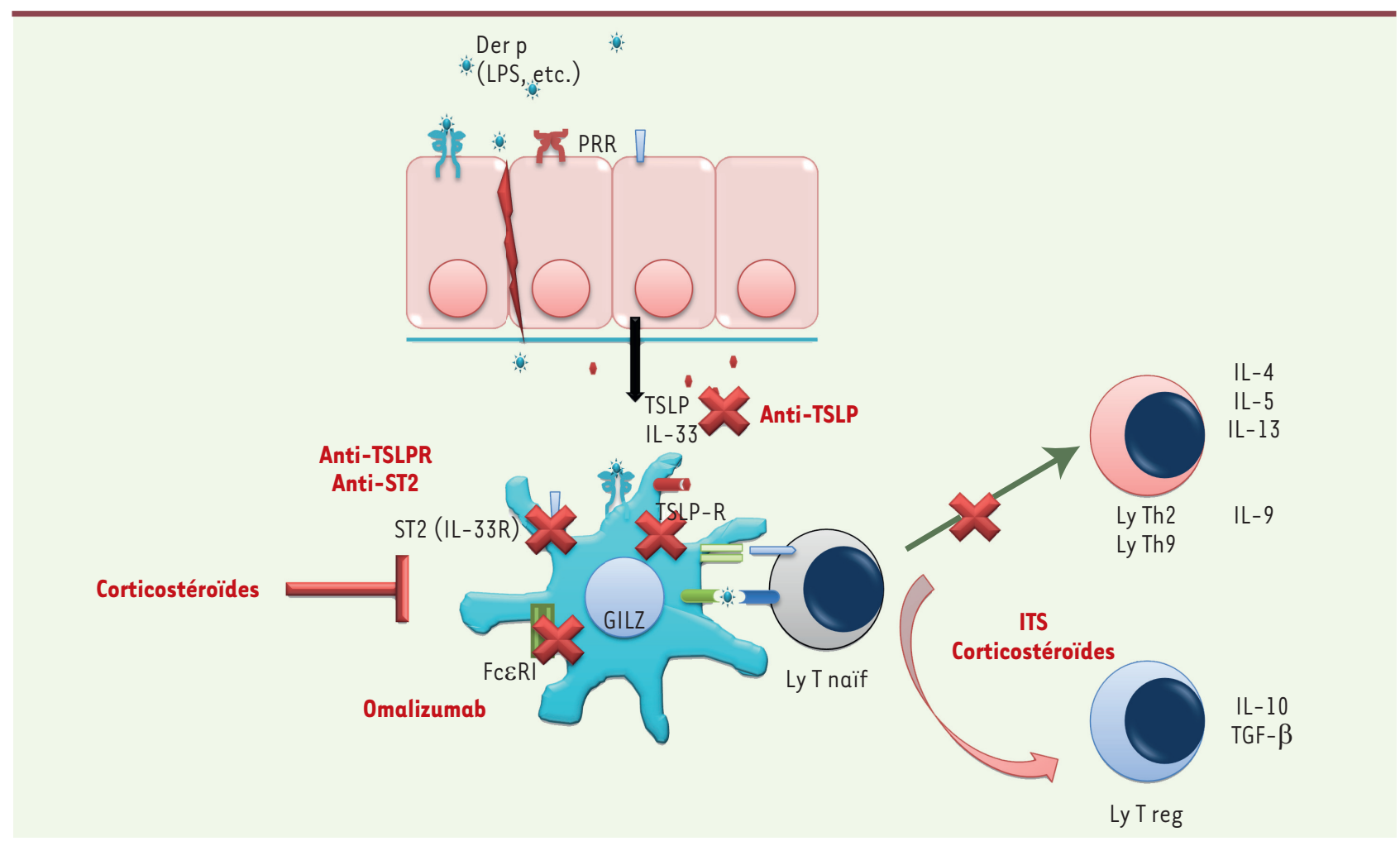

Figure 4. Synthèse des cibles thérapeutiques offertes par les $C D$ et leur micro-environnement. Les corticostéroïdes diminuent le nombre de CD au niveau épithélial et favorisent la génération de lymphocytes T regulateurs (Ly T reg) via leur action sur GILZ. L'immunothérapie spécifique favorise également l'expansion de Ly T reg via son action sur les CD. L'omalizumab diminue l'expression du FcERI à la surface des CD et empêche ainsi la présentation facilitée des allergènes. Des anticorps ciblant la TSLP, son récepteur ou un des récepteurs de l'IL-33 (ST2) ont donné des résultats précliniques encourageants.

\section{SUMMARY}

Human dendritic cells in allergic asthma and rhinitis

The role of dendritic cells (DC) in the pathogenesis of allergic asthma and rhinitis has been highlighted for 15 years. In this review, we summarize key findings concerning DC function in airway allergy and focus on studies performed in human. DC coming from allergic patients have specific characteristics, including significant expression of high affinity receptor for $\lg \varepsilon$ as well as a propensity for Th2 responses induction following priming with allergen and/or epithelial cytokines. Mechanistic data concerning this DC dysfunction in asthma also provide perspectives for innovating therapies. $\diamond$

\section{REMERCIEMENTS}

A. Froidure est financé par la fondation Saint-Luc-cliniques universitaires Saint-Luc (Belgique) et la fondation de Vooght-université catholique de Louvain (Belgique). C. Pilette est spécialiste post-doctorant du Fonds national pour la recherche scientifique (FNRS 1.R.016.14) et du Walloon institute for excellence in lifesciences and biotechnology (WELBIO CR-2012S-05). Ce travail est soutenu par une bourse (FRSM 3.4522.12) du Fonds de la recherche scientifique (FNRS), ainsi que par une bourse de recherche de GSK.

\section{LIENS D'INTÉREิT}

Les auteurs déclarent n'avoir aucun lien d'intérêt concernant les données publiées dans cet article.

\section{RÉFÉRENCES}

1. Global initiative for asthma (GINA). Burden Report 2004. Available at: www. ginasthma.org.

2. Bousquet J, Khaltaev $N$, Cruz AA, et al. Allergic rhinitis and its impact on asthma (ARIA) 2008 update (in collaboration with the World health organization, GA(2)LEN and AllerGen). Allergy $2008 ; 63$ (suppl 86) : 8-160.

3. Barnes PJ. Pathophysiology of allergic inflammation. Immunol Rev 2011 ; $242: 31-50$.

4. Soroosh P, Doherty TA. Th9 and allergic disease. Immunology $2009 ; 127: 450-8$.

5. Cosmi L, Liotta F, Maggi $\varepsilon$, et al. Th17 cells: new players in asthma pathogenesis. Allergy 2011 ; 66 : 989-98.

6. Lambrecht $B N$, Hammad $H$. The role of dendritic and epithelial cells as master regulators of allergic airway inflammation. Lancet $2010 ; 376: 835-43$.

7. Steinman RM, Cohn ZA. Identification of a novel cell type in peripheral lymphoid organs of mice. I. Morphology, quantitation, tissue distribution. J Exp Med 1973; $137: 1142-62$.

8. Zhou LJ, Tedder TF. CD14 $4^{+}$blood monocytes can differentiate into functionally mature CD83+ dendritic cells. Proc Natl Acad Sci USA 1996 ; 93 : 2588-92.

9. Reizis B, Bunin A, Ghosh HS, et al. Plasmacytoid dendritic cells: recent progress and open questions. Annu Rev Immunol 2011 ; 29 : 163-83.

10. Segura $\varepsilon$, Amigorena $S$. Inflammatory dendritic cells in mice and humans. Trends Immunol $2013 ; 34: 440-5$.

11. Plantinga M, Guilliams M, Vanheerswynghels $M$, et al. Conventional and monocytederived CDIl $b^{+}$dendritic cells initiate and maintain Thelper 2 cell-mediated immunity to house dust mite allergen. Immunity $2013 ; 38$ : 322-35.

12. Demedts IK, Brusselle GG, Vermaelen KY, Pauwels RA. Identification and characterization of human pulmonary dendritic cells. Am J Respir Cell Mol Biol $2005 ; 32$ : 177-84.

13. Haniffa M, Shin A, Bigley V, et al. Human tissues contain CD14lhi crosspresenting dendritic cells with functional homology to mouse $\mathrm{CD} 103^{+}$ nonlymphoid dendritic cells. Immunity $2012 ; 37: 60-73$. 


\section{RÉFÉRENCES}

14. Segura $\varepsilon$, Touzot $M$, Bohineust $A$, et al. Human inflammatory dendritic cells induce Th17 cell differentiation. Immunity $2013 ; 38: 336-48$.

15. Andersson LI, Cirkic $\varepsilon$, Hellman P, Eriksson H. Myeloid blood dendritic cells and monocyte-derived dendritic cells differ in their endocytosing capability. Hum Immunol $2012 ; 73: 1073-81$.

16. Lambrecht BN, Salomon B, Klatzmann D, Pauwels RA. Dendritic cells are required for the development of chronic eosinophilic airway inflammation in response to inhaled antigen in sensitized mice. J Immunol $1998 ; 160: 4090-7$

17. De Heer HJ, Hammad H, Soullie T, et al. Essential role of lung plasmacytoid dendritic cells in preventing asthmatic reactions to harmless inhaled antigen. J Exp Med $2004 ; 200$ : 89-98.

18. Nakano H, Free ME, Whitehead GS, et al. Pulmonary CD $103^{+}$dendritic cells prime Th2 responses to inhaled allergens. Mucosal Immunol $2012 ; 5: 53-65$.

19. Hammad H. Th2 polarization by Der $\mathrm{p} 1$-pulsed monocyte-derived dendritic cells is due to the allergic status of the donors. Blood $2001 ; 98: 1135-41$.

20. Charbonnier AS. Der p 1-pulsed myeloid and plasmacytoid dendritic cells from house dust mitesensitized allergic patients dysregulate the T cell response. J Leukoc Biol $2003 ; 73$ : 91-9.

21. Farrell $\varepsilon, O^{\prime}$ Connor TM, Duong M, et al. Circulating myeloid and plasmacytoid dendritic cells after allergen inhalation in asthmatic subjects. Allergy $2007 ; 62$ : 1139-45.

22. Dua B, Watson RM, Gauvreau GM, O’Byrne PM. Myeloid and plasmacytoid dendritic cells in induced sputum after allergen inhalation in subjects with asthma. J Allergy Clin Immunol $2010 ; 126$ : $133-9$.

23. Upham JW, Denburg JA, O'Byrne PM. Rapid response of circulating myeloid dendritic cells to inhaled allergen in asthmatic subjects. Clin Exp Allergy $2002 ; 32: 818-23$.

24. Dua B, Smith S, Kinoshita T, et al. Myeloid dendritic cells type 2 in allergic asthma. Allergy 2013 ; $68: 1322-6$.

25. Dua B, Tang W, Watson R, et al. Myeloid dendritic cells type 2 after allergen inhalation in asthmatic subjects. Clin Exp Allergy $2014 ; 44$ : 921-9.

26. Foster B, Metcalfe DD, Prussin C. Human dendritic cell 1 and dendritic cell 2 subsets express FcepsilonRI: correlation with serum Ig $\varepsilon$ and allergic asthma. J Allergy Clin Immunol 2003 ; 112 : 1132-8.

27. Maurer D, Fiebiger S, Ebner $\mathrm{C}$, et al. Peripheral blood dendritic cells express $\mathrm{Fc}$ epsilon RI as a complex composed of Fc epsilon RI alpha- and Fc epsilon RI gamma-chains and can use this receptor for IgE-mediated allergen presentation. J Immunol 1996; 157 : 607-16.

28. Greer AM, Wu N, Putnam AL, et al. Serum Ig clearance is facilitated by human FcepsilonRI internalization. J Clin Invest $2014 ; 124$ : 1187-98.

29. Shen C, Hupin C, Froidure A, et al. Impaired ICOSL in human myeloid dendritic cells promotes Th2 responses in patients with allergic rhinitis and asthma. Clin Exp Allergy $2014 ; 44: 831-41$.

30. Pilette C, Jacobson MR, Ratajczak C, et al. Aberrant dendritic cell function conditions Th2-cell polarization in allergic rhinitis. Allergy $2013 ; 68: 312-21$.

31. Platts-Mills TA, Woodfolk JA. Allergens and their role in the allergic immune response. Immunol Rev $2011 ; 242: 51-68$.

32. Hammad H, Chieppa M, Perros F, et al. House dust mite allergen induces asthma via Toll-like receptor 4 triggering of airway structural cells. Nat Med $2009 ; 15: 410-6$.

33. Ito $\mathrm{T}$, Wang $\mathrm{YH}$, Duramad 0 , et al. TSLP-activated dendritic cells induce an inflammatory Thelper type 2 cell response through 0 X40 ligand. J Exp Med $2005 ; 202$ : 1213-23.

34. Shikotra A, Choy DF, Ohri CM, et al. Increased expression of immunoreactive thymic stromal lymphopoietin in patients with severe asthma. J Allergy Clin Immunol 2012; 129 : 104-11 el-9.

35. Jiang H, Hener P, Li J, Li M. Skin thymic stromal lymphopoietin promotes airway sensitization to inhalant house dust mites leading to allergic asthma in mice. Allergy $2012 ; 67: 1078-82$.

36. Xu G, Zhang L, Wang DY, et al. Opposing roles of IL-17A and IL-25 in the regulation of TSLP production in human nasal epithelial cells. Allergy $2010 ; 65: 581-9$.

37. Zhu Z, Oh MH, Yu J, et al. The role of TSLP in IL-13-induced atopic march. Sci Rep 2011; 1 : 23.

38. Froidure A, Shen C, Gras D, et al. Myeloid dendritic cells are primed in allergic asthma for thymic stromal lymphopoietin-mediated induction of Th2 and Th9 responses. Allergy $2014 ; 69: 1068-76$

39. Lambrecht BN, Hammad H. Asthma: the importance of dysregulated barrier immunity. Eur J Immunol 2013; $43: 3125-37$.
40. Chu DK, Llop-Guevara A, Walker TD, et al. IL-33, but not thymic stromal lymphopoietin or IL-25, is central to mite and peanut allergic sensitization. J Allergy Clin Immunol 2013 ; 131 : 187-200 el-8.

41. Willart MA, Deswarte K, Pouliot $P$, et al. Interleukin-lalpha controls allergic sensitization to inhaled house dust mite via the epithelial release of GM-CSF and IL-33.J Exp Med $2012 ; 209$ : 1505-17.

42. Barnes PJ. Efficacy of inhaled corticosteroids in asthma. J Allergy Clin Immunol $1998 ; 102: 531-8$.

43. Moller GM, Overbeek SE, Van Helden-Meeuwsen CG, et al. Increased numbers of dendritic cells in the bronchial mucosa of atopic asthmatic patients: downregulation by inhaled corticosteroids. Clin Exp Allergy 1996 ; 26 : 517-24.

44. Krzysiek R. Role of glucocorticoid-induced leucine zipper (GILZ) expression by dendritic cells in tolerance induction. Transplant Proc $2010 ; 42: 3331-2$

45. Humbert M, Beasley R, Ayres J, et al. Benefits of omalizumab as add-on therapy in patients with severe persistent asthma who are inadequately controlled despite best available therapy (GINA 2002 step 4 treatment): INNOVATE. Allergy $2005 ; 60: 309-16$.

46. Chanez P, Contin-Bordes C, Garcia G, et al. Omalizumab-induced decrease of FcxiRl expression in patients with severe allergic asthma. Respir Med 2010 ; $104: 1608-17$.

47. Schroeder JT, Bieneman AP, Chichester KL, et al. Decreases in human dendritic cell-dependent $\mathrm{T}(\mathrm{H}) 2$-like responses after acute in vivo Ig $\varepsilon$ neutralization. J Allergy Clin Immunol 2010 ; 125 : 896-901.

48. Akdis M, Akdis CA. Mechanisms of allergen-specific immunotherapy: multiple suppressor factors at work in immune tolerance to allergens. J Allergy Clin Immunol 2014 ; $133: 621-31$.

49. Chen ZG, Zhang $T$, Li HT, et al. Neutralization of TSLP inhibits airway remodeling in a murine model of allergic asthma induced by chronic exposure to house dust mite. PLoS One 2013; 8 : e51268.

50. Cheng DT, Ma C, Niewoehner J, et al. Thymic stromal lymphopoietin receptor blockade reduces allergic inflammation in a cynomolgus monkey model of asthma. J Allergy Clin Immunol 2013 ; 132 : 455-62.

51. Lee HY, Rhee CK, Kang JY, et al. Blockade of IL-33/ST2 ameliorates airway inflammation in a murine model of allergic asthma. Exp Lung Res $2014 ; 40$ 66-76.

52. Gauvreau GM, O'Byrne PM, Boulet LP, et al. Effects of an anti-TSLP antibody on allergen-induced asthmatic responses. N Engl J Med $2014 ; 370: 2102-10$.

53. Zitvogel L, Amigorena S, Teillaud JL. À propos de Ralph M. Steinman et des cellules dendritiques. Prix Nobel de Médecine 2011. Med Sci (Paris) 2011 $27: 1028-34$

54. Crozat K, Dalod M. Les cellules dendritiques humaines exprimant BDCA3. Med Sci (Paris) $2011 ; 27: 25-8$.

55. Henri S, Poulin L, Malissen B. Le derme de la peau s'enrichit d'une nouvelle population de cellules dendritiques. Med Sci (Paris) 2008 ; $24: 346-7$.

56. Segura $\varepsilon$, Amigorena S. Les cellules dendritiques inflammatoires. Med Sci (Paris) $2014 ; 30: 64-8$.

57. Pépin $A$, Biola-Vidamment $A$, Latré de Laté $P$, et al. Les protéines de la famille TSC-22D : de nouveaux régulateurs de l'homéostasie cellulaire? Med Sci (Paris) $2015 ; 31: 75-83$.

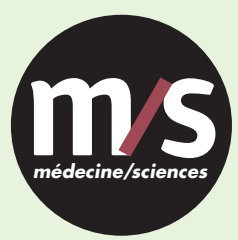

Tarifs d'abonnement $m / s-2015$

Abonnez-vous

à médecine/sciences
$>$ Grâce à $\mathrm{m} / \mathrm{s}$, vivez en direct les progrès des sciences biologiques et médicales

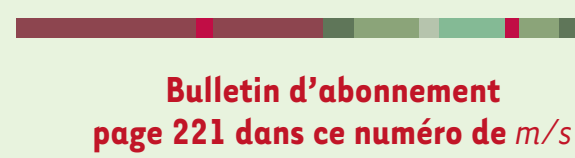

TIRÉS À PART

A. Froidure

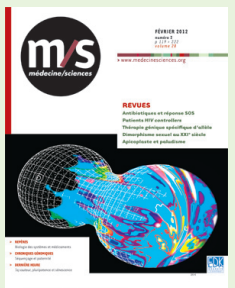

